\title{
Fiber Path Optimization of Symmetric Laminates with Cutouts for Thermal Buckling
}

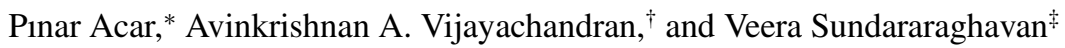 \\ University of Michigan, Ann Arbor, Michigan 48109 \\ and \\ Anthony M. Waas $\underline{\underline{s}}$ \\ University of Washington, Seattle, Washington 98195-2400 \\ DOI: $10.2514 / 1 . C 033866$
}

\begin{abstract}
Automated fiber placement technology has pushed for the need to explore nonconventional fiber paths in laminated composites. This paper investigates optimal spatially varying fiber paths in a symmetric linear orthotropic laminate, which could increase the critical buckling temperature under uniform applied thermal loads. The key idea here is to achieve gains in buckling performance yet focus on manufacturability of the obtained optimal fiber path. The subject of this study is a four-layer symmetric orthotropic laminated plate, with a central circular cutout that is clamped on all the edges. A novel finite element algorithm is proposed, which imposes a condition on the definition of discrete fiber angles within each element. The main objectives of the proposed finite element approach are to maintain continuity of the fiber paths and to use a computationally efficient model by reducing the number of optimization variables.
\end{abstract}

\section{Nomenclature}

$B_{1}, B_{2}=$ first- and second-order derivatives of shape (interpolation) functions

$C=$ upper-bound averages along thickness, of the constitutive matrices in the fiber axis

$D=$ finite element material matrix

$\mathrm{d} \theta=$ variation in the fiber angle across finite elements

$h=$ thickness of the lamina

$K_{b}, K_{g}=$ elastic and geometric stiffnesses

$n=$ number of elements in cycloidal direction

$Q, \bar{Q}=$ constitutive matrices in the global and fiber axes, respectively

$T(m)=$ fiber angle within any finite element $m$

$T_{i, p}^{q} \quad=\quad$ initial fiber angle for the $p$ th quadrant in the $q$ th layer

$u, v, w=$ displacements in the $x, y$, and $z$ directions

$z_{q} \quad=$ lamina thickness for the $q$ th layer

$\alpha=$ coefficients of thermal expansion

$\Delta T_{\mathrm{cr}} \quad=\quad$ critical buckling temperature

\section{Introduction}

C OMPOSITE platelike components with cutouts are very common in aerospace structures due to their high strength-toweight and high stiffness-to-weight properties. They are used as access ports for mechanical and electrical systems, lightening holes to reduce the total mass of structural parts, and even as windows in the fuselage. For example, cutouts are used in wing spars and ribs as well as the interior hull of the fuselage, as both accesses to control lines

Presented as Paper 2016-1725 at the 57th AIAA/ASCE/AHS/ASC Structures, Structural Dynamics, and Materials Conference, San Diego, CA, 04-08 January 2016; received 21 January 2016; revision received 29 February 2016; accepted for publication 7 March 2016; published online 30 May 2016. Copyright (C) 2016 by Pinar Acar. Published by the American Institute of Aeronautics and Astronautics, Inc., with permission. Copies of this paper may be made for personal and internal use, on condition that the copier pay the percopy fee to the Copyright Clearance Center (CCC). All requests for copying and permission to reprint should be submitted to CCC at www.copyright.com; employ the ISSN 0021-8669 (print) or 1533-3868 (online) to initiate your request.

*Graduate Research Assistant, Department of Aerospace Engineering. Student Member AIAA.

${ }^{\dagger}$ Graduate Research Assistant, Department of Aerospace Engineering.

${ }^{\ddagger}$ Associate Professor, Department of Aerospace Engineering. Member AIAA.

${ }^{\S}$ Boeing-Egtvedt Chair, Department of Aeronautics and Astronautics. Member AIAA. and structural inspections, as well as reducing the mass of these members. Their use as backing structures for space mirrors is made possible with the advent of advanced fiber placement machines [1], which are currently being used in the manufacturing of large aerospace structures. The structures with cutouts usually experience elevated compressive mechanical and thermal loads, which can result in buckling. Therefore, the design phase of these structures requires consideration of buckling performance.

Effects of cutouts on strength and buckling of thin plates and thinwalled cylindrical shells have been presented by various researchers. Kirsch [2], in 1898, first proposed the exact closed-form analytical solutions for the stress concentrations around cutouts in an infinite isotropic lamina under uniaxial tension. Mansfield [3] proposed methods to restore the stress state of an isotropic plate with a cutout to that of one without, thereby reducing any stress concentrations that could cause fracture. Pipes et al. [4] presented a study on notched strength of composite materials, whereas Tang [5] proposed studies on interlaminar stresses in orthotropic plates with a cutout, under tension. Ahn and Waas [6] provided experimental results and a novel mechanism-based modeling approach to predict the compressive notched strength of laminates under uniaxial and biaxial loading. Senocak and Waas $[7,8]$ revisited the problem of a uniaxial tensile loaded plate with a cutout in the case of an orthotropic laminate and proposed methods to essentially nullify the stress concentrations around the cutouts, by duly considering the bending-stretching coupling and using stiffeners modeled as one-dimensional fourdegree-of-freedom beams. Acar et al. [9] presented an algorithm for optimal steered fiber paths to reduce stress concentrations around cutouts in a symmetric laminate. Lin et al. [10] presented buckling models for laminated plates with holes, and Topal and Uzman [11] studied the maximization of buckling loads for laminated plates with cutouts. Ounis et al. [12] performed a finite-element-based study to analyze thermal buckling of laminated composites. A method to minimize the thermal expansion of laminates is presented by Rangarajan et al. [13]. Hyer and Lee [14] studied a problem on the buckling resistance of composite plates with central circular holes in the case of curvilinear fibers and proposed a solution based on modeling individual fiber orientations within each finite element (FE), where the fiber orientations are allowed to vary spatially within the lamina. Gurdal and Olmedo [15] presented an optimization of spatially varying fiber paths for a solution to a plane elasticity problem using a variable-stiffness concept.

The subject of interest in this paper is to derive optimal spatially varying fiber paths and layer thicknesses for a four-layer symmetric orthotropic composite laminate with the central circular cutout to improve the thermal buckling performance. The square plate is 
clamped on all the edges and is under uniform thermal loads. A novel finite element algorithm is proposed, which ensures that the variations in fiber angles across adjacent finite elements pick discrete values and thus maintain a constant fiber angle within each element, making it easy to obtain a smooth fiber path. This model can easily be reproduced for a laminate with as many layers following the same procedure. The problem formulation accounts for this fiber path definition to solve the critical buckling temperature. The multiobjective optimization is performed to identify the fiber path that maximizes the critical buckling temperature and minimizes the mass of the laminate.

This work aims to increase the buckling performance of a composite plate structure with a cutout by optimizing the fiber angle paths. The structures with these optimal steered fiber paths provide better resistance to thermal buckling even in elevated temperatures. The organization of the paper is as follows. Section II introduces the composite plate thermal buckling problem. In Sec. III, the proposed meshing algorithm for fiber continuity and finite element methodology for the solution are discussed. The finite element solution is verified in Sec. IV. Section $\underline{\mathrm{V}}$ provides information about the optimization problem and optimum results. Section VI concludes the paper with a discussion of future extensions of this study.

\section{Problem Definition}

The present work comprises thermal buckling of a clamped plate under the effect of thermal axial loads. The plate is modeled as a symmetric four-layer composite laminate. The problem is separated into two parts; the first part explains the algorithm and the finite element procedure, whereas the second explains the optimization

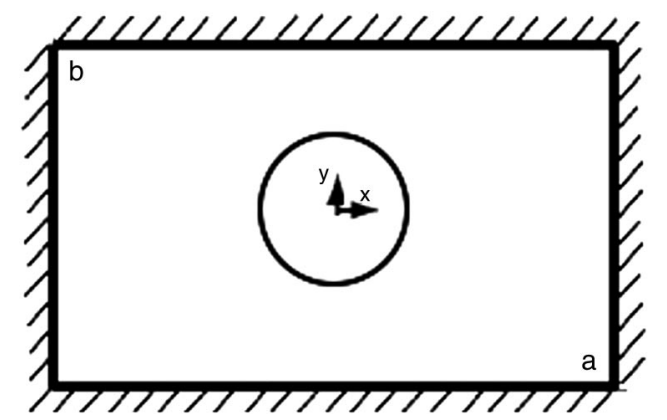

Fig. 1 Clamped plate with a cutout under uniform heating. problem setup. The loading and geometry are symmetric because the entire plate is under a uniform thermal load, inducing axial compressive and shear loads on the plate, described as $N_{x}, N_{y}$, and $N_{x y}$ in Fig. 1. Even though the symmetry of the loading and geometry suggests the use of symmetry boundary conditions to obtain the prebuckling stresses by analyzing only one quarter of the plate, it needs to be noted that the buckling modes need not be symmetric. By using symmetry boundary conditions, only the symmetric modes can be obtained. Therefore, the full plate model is modeled to obtain all the buckling modes. The critical buckling temperature is then solved for the first buckling mode.

\section{Meshing Algorithm}

The proposed finite element meshing method has multiple features. First, it always ensures continuity of the fiber paths across element boundaries. Second, it is computationally efficient because only four optimization variables per layer of the laminate are used to define the fiber path. Moreover, it could easily be scaled for any number of plies in any symmetric or asymmetric laminate. The algorithm explains how discrete fiber angles are computed for each finite element, ensuring interelement continuity of the fiber angle. This is essential to end up with a manufacturable design using automated fiber placement (AFP) technology.

\section{A. Algorithm for Discrete Fiber Angles}

This section discusses the algorithm to compute a discrete fiber angle $T(m)$ for each finite element under prescribed conditions of fiber path continuity. The direction of $m$ (where $0<m \leq n$, with $n$ being the total number of elements in cycloidal direction) represents the count of the finite element along each row. The plate is divided into four subdomains (quadrants) to ensure fiber path continuity (Fig. 2). In the context of spatially varying fiber paths, symmetry in the laminate is described by the same spatial variation of fiber paths in the layers symmetric with respect to the center plane. In this specific case, layer 1 is the same as layer 4 , and layer 2 is the same as layer 3 . Detailed later is the formulation of the algorithm for the plate.

For the elements located in the $p$ th quadrant and in the $q$ th layer, $T_{i, p}^{q}$ and $T_{f, p}^{q}$ represent initial and final fiber angles, respectively (e.g., $T_{i, 1}^{1}$ and $T_{f, 1}^{1, p}$ are initial and final fiber angles for the first quadrant in layer 1). Hence, the change in fiber angle is defined across each finite element along the direction ' $m$ ' for layer $1, d \theta_{1}^{1}$, as follows:

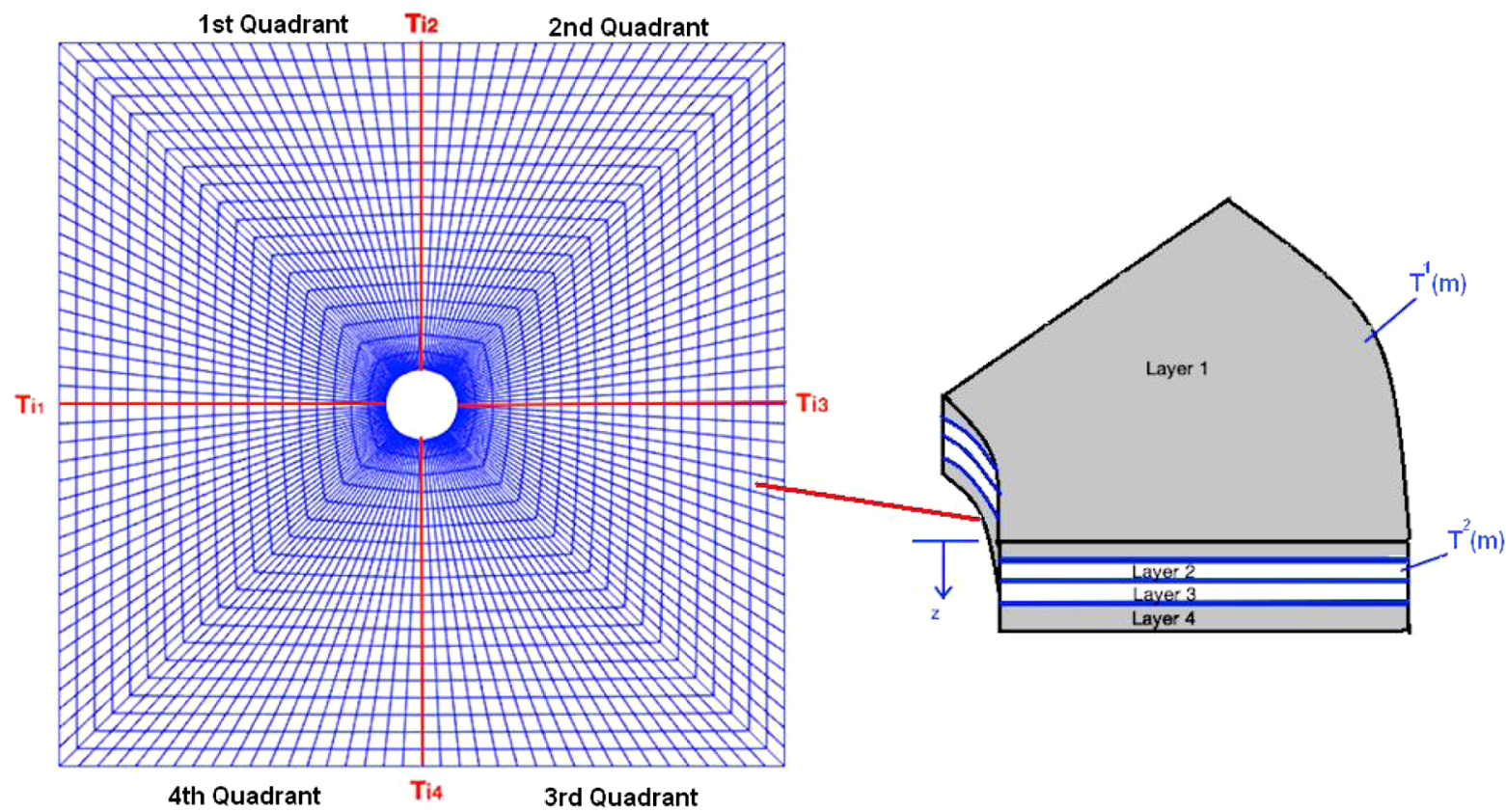

Fig. 2 Finite element mesh for quarter plate ( 2888 plate elements). 


$$
d \theta_{1}^{1}=\frac{T_{f, 1}^{1}-T_{i, 1}^{1}}{n-1}
$$

To elaborate, for any $q$ th layer, $T_{i, 1}^{q}, T_{i, 2}^{q}, T_{i, 3}^{q}$, and $T_{i, 4}^{q}$ are the "initial" fiber angles of quadrants $1,2,3$, and 4 . Then, the final fiber angles for these quadrants are noted as $T_{f, 1}^{q}=T_{i, 2}^{q}, T_{f, 2}^{q}=T_{i, 3}^{q}$, $T_{f, 3}^{q}=T_{i, 4}^{q}$, and $T_{f, 4}^{q}=T_{i, 1}^{q}$, which merely means that the final fiber angle of each quadrant becomes the initial fiber angle of the next quadrant to ensure continuity of fiber angles. Hence, the discrete variation in angle in each quadrant $d \theta_{p}^{q}$ for each $p$ th quadrant for the $q$ th layer is obtained as

$$
d \theta_{p}^{q}=\frac{T_{f, p}^{q}-T_{i, p}^{q}}{n-1}
$$

This would mean that the only condition imposed on each $T_{i, p}^{q}$ during optimization on its range is $\left(0 \leq T_{i, 1}^{q}, \quad T_{i, 2}^{q}, \quad T_{i, 3}^{q}\right.$, $T_{i, 4}^{q}<180 \mathrm{deg}$ ), which constrains the fiber angle inside each element, $T(m)$. By means of constraining the value of $d \theta_{p}^{q}$ and thereby imposing a specific $T(m)$ for each element $m$, the essence of the solution is captured, viz., to enforce continuity of the fiber angle, making it easily adaptable for manufacturing. The fiber angle in each element in the $p$ th quadrant for the $q$ th layer as it moves along direction ' $m$ ' in Fig. 2 is given by the expression

$$
T_{p}(m)^{q}=T_{i, p}^{q}+(m-1) d \theta_{p}^{q}
$$

where $m(0<m \leq n)$ represents the element number in the chosen cycloidal direction in Fig. 2 , and $n$ is the maximum number of elements in this direction. As will be discussed in the next section, the fiber angle $T(m)$ specified for each element under restrictions arising from $d \theta$ would be used in the finite element formulation by effectively computing the finite element material matrix $D$ using the plane stress constitutive matrix $\bar{Q}$ averaged over the thickness to obtain an upper bound matrix $C$. $\bar{Q}$ is obtained by rotating the constitutive matrix $Q$ by a fiber angle $T(m)$ for each element.

\section{B. Finite Element Model}

When heated uniformly, the thermal expansion of the restricted plates will result in uniformly distributed in-plane normal and shear forces (Fig. 1). The constitutive matrix $Q$ of each finite element depends on the particular fiber angle within that finite element. The regular finite element procedure starts with the computation of the constitutive matrix in the global frame $Q_{i j}$ (where $i, j=1,2,3,4,5$, 6 ). However, the problem needs to be assembled and solved in the material frame. This means that the material matrix in the material frame $\bar{Q}_{i j}$ can be computed for each finite element with the transformation through an angle $T(m)$. The stress-strain relation in the global frame for a general three-dimensional orthotropic material is

$$
\left[\begin{array}{c}
\sigma_{x x} \\
\sigma_{y y} \\
\sigma_{z z} \\
\sigma_{z x} \\
\sigma_{y z} \\
\tau_{x y}
\end{array}\right]=\left[\begin{array}{cccccc}
Q_{11} & Q_{12} & Q_{13} & 0 & 0 & 0 \\
Q_{12} & Q_{22} & Q_{23} & 0 & 0 & 0 \\
Q_{13} & Q_{23} & Q_{33} & 0 & 0 & 0 \\
0 & 0 & 0 & Q_{44} & 0 & 0 \\
0 & 0 & 0 & 0 & Q_{55} & 0 \\
0 & 0 & 0 & 0 & 0 & Q_{66}
\end{array}\right]\left[\begin{array}{c}
\epsilon_{x x} \\
\epsilon_{y y} \\
\epsilon_{z z} \\
\gamma_{z x} \\
\gamma_{y z} \\
\gamma_{x y}
\end{array}\right]
$$

Table 1 Material and thermal properties of IM7/8551-7

\begin{tabular}{lc}
\hline \hline Parameter & Value \\
\hline$E_{x x}$ & $162.0 \mathrm{GPa}$ \\
$E_{y y}$ & $8.34 \mathrm{GPa}$ \\
$G_{x y}$ & $2.07 \mathrm{GPa}$ \\
$G_{y z}$ & $2.07 \mathrm{GPa}$ \\
$\nu_{x y}$ & 0.339 \\
$\nu_{y z}$ & 0.509 \\
$\alpha_{x x}$ & $-0.48 \mu \mathrm{m} / \mathrm{m} /{ }^{\circ} \mathrm{C}$ \\
$\alpha_{y y}$ & $22.3 \mu \mathrm{m} / \mathrm{m} /{ }^{\circ} \mathrm{C}$ \\
\hline \hline
\end{tabular}
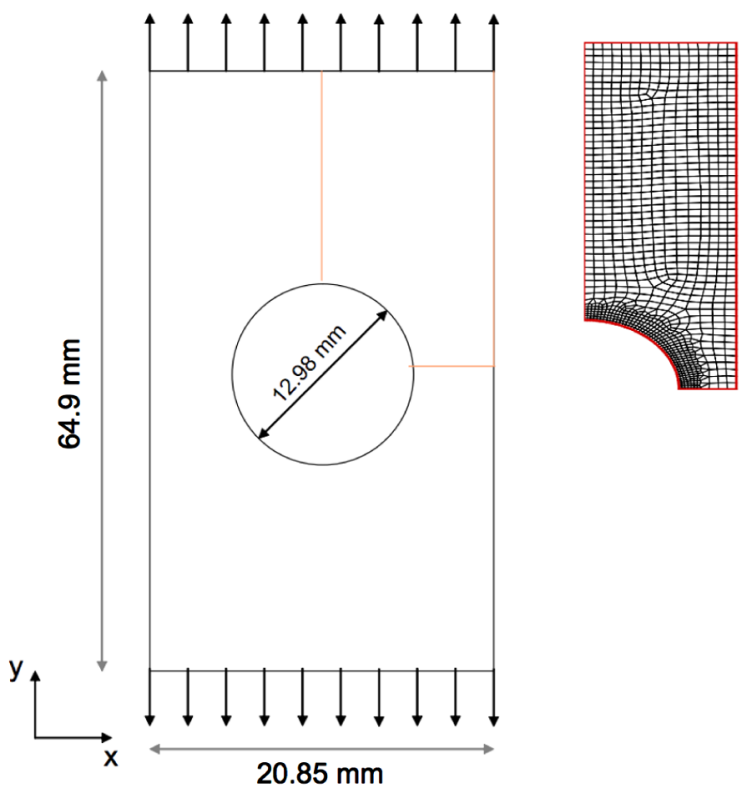

Fig. 3 Problem definition for fringe contour comparison.

where the stiffness coefficients are functions of the orthotropic material properties $E_{11}, E_{22}, \nu_{12}, \nu_{23}$, and $G_{23}$. The material and thermal properties used in this work correspond to that of IM7/8551-7 [16,17] and are detailed in Table 1 . Note that extension and shear are uncoupled.

To assemble the global stiffness matrices $\left[K_{b}\right]$ and $\left[K_{g}\right]$, the stiffness of each finite element is to be calculated in the respective material coordinate systems and then assembled. For each finite element, a transformation matrix $[L]=f(T(m))$ is calculated as

$$
[L]=\left[\begin{array}{cccccc}
1 & 0 & 0 & 0 & 0 & 0 \\
0 & c_{p}^{2} & s_{p}^{2} & 2 c_{p} s_{p} & 0 & 0 \\
0 & s_{p}^{2} & c_{p}^{2} & -2 c_{p} s_{p} & 0 & 0 \\
0 & -c_{p} s_{p} & c_{p} s_{p} & c_{p}^{2}-s_{p}^{2} & 0 & 0 \\
0 & 0 & 0 & 0 & c_{p} & -s_{p} \\
0 & 0 & 0 & 0 & s_{p} & c_{p}
\end{array}\right]
$$

where $c_{p}=\cos (T(m))$ and $s_{p}=\sin (T(m))$ for each element ' $m$ '. The rotated constitutive matrix in the material frame for each element ' $m$ ', $\bar{Q}_{i j}(m)$, is obtained as

$$
\bar{Q}_{i j}=[L]^{T}[Q][L]
$$

Because the finite element formulation is basically dealing with a multilayer structure in this case, and each finite element was previously defined through the thickness of the whole structure, the material matrix should be averaged along the thickness of each particular element (Fig. 3) to obtain an upper-bound averaged material matrix $C$. This averaging along the thickness indicates the upper-bound approach to compute $\bar{Q}_{i j}$ with the assumption of strain continuity through the thickness of the element. If $z_{1}$ and $z_{2}$ are the thicknesses of the first (and fourth) layers and the second (and third) layers, respectively, then $\bar{Q}_{i j}$ elements of the multilayer structure can be obtained as an upper bound average [18]:

$$
\bar{Q}_{i j, \text { avg }}=\frac{z_{1} \bar{Q}_{i j}^{1}+z_{2} \bar{Q}_{i j}^{2}}{z_{1}+z_{2}}
$$

where $i, j=1,2,3,4,5,6$, for any element of the averaged $\bar{Q}_{\mathrm{avg}}$ matrix: 


$$
\begin{aligned}
{[C] } & =\left[\bar{Q}_{\text {avg }}\right] \\
& =\left[\begin{array}{cccccc}
\bar{Q}_{11, \text { avg }} & \bar{Q}_{12, \text { avg }} & \bar{Q}_{13 \text { avg }} & 0 & 0 & 0 \\
\bar{Q}_{12, \text { avg }} & \bar{Q}_{22, \text { avg }} & \bar{Q}_{23, \text { avg }} & 0 & 0 & 0 \\
\bar{Q}_{13, \text { avg }} & \bar{Q}_{23, \text { avg }} & \bar{Q}_{33 \text {,avg }} & 0 & 0 & 0 \\
0 & 0 & 0 & \bar{Q}_{44, \text { avg }} & 0 & 0 \\
0 & 0 & 0 & 0 & \bar{Q}_{55 \text {,avg }} & 0 \\
0 & 0 & 0 & 0 & 0 & \bar{Q}_{66, \text { avg }}
\end{array}\right]
\end{aligned}
$$

where $C$ is the elastic stiffness matrix of each particular element. The flexural rigidities $D$ can be obtained from the elastic stiffness matrix, using plane stress assumptions, by the following relation [19]:

$$
\begin{aligned}
& {\left[\begin{array}{lll}
D_{11} & D_{12} & D_{13} \\
D_{21} & D_{22} & D_{23} \\
D_{31} & D_{32} & D_{33}
\end{array}\right]=\frac{h^{3}}{12} \times\left(\left[\begin{array}{lll}
C_{11} & C_{12} & C_{16} \\
C_{21} & C_{22} & C_{26} \\
C_{61} & C_{62} & C_{66}
\end{array}\right]-\left[\begin{array}{lll}
C_{13} & C_{14} & C_{15} \\
C_{23} & C_{24} & C_{25} \\
C_{63} & C_{64} & C_{65}
\end{array}\right]\right.} \\
& \left.\times\left(\left[\begin{array}{lll}
C_{33} & C_{34} & C_{35} \\
C_{43} & C_{44} & C_{45} \\
C_{53} & C_{54} & C_{55}
\end{array}\right]\right)^{-1} \times\left[\begin{array}{lll}
C_{31} & C_{32} & C_{36} \\
C_{41} & C_{42} & C_{46} \\
C_{51} & C_{52} & C_{56}
\end{array}\right]\right)
\end{aligned}
$$

The general classical buckling equations for a rectangular laminated plate with dimensions $a \times b \times h$ (length in the $x$ direction $\times$ length in the $y$ direction $\times$ height) subjected to uniform temperature rise are

$$
\begin{gathered}
A_{11} \frac{\partial^{2} u_{0}}{\partial x^{2}}+2 A_{16} \frac{\partial^{2} u_{0}}{\partial x \partial y}+A_{66} \frac{\partial^{2} u_{0}}{\partial y^{2}}+A_{16} \frac{\partial^{2} v_{0}}{\partial x^{2}} \\
+\left(A_{12}+A_{66}\right) \frac{\partial^{2} v_{0}}{\partial x \partial y}+A_{26} \frac{\partial^{2} v_{0}}{\partial y^{2}}=0
\end{gathered}
$$

$$
\begin{aligned}
& A_{16} \frac{\partial^{2} u_{0}}{\partial x^{2}}+\left(A_{12}+A_{66}\right) \frac{\partial^{2} u_{0}}{\partial x \partial y}+A_{26} \frac{\partial^{2} u_{0}}{\partial y^{2}}+A_{66} \frac{\partial^{2} v_{0}}{\partial x^{2}} \\
& +2 A_{26} \frac{\partial^{2} v_{0}}{\partial x \partial y}+A_{22} \frac{\partial^{2} v_{0}}{\partial y^{2}}=0
\end{aligned}
$$

$$
\begin{aligned}
& D_{11} \frac{\partial^{4} w}{\partial x^{4}}+4 D_{16} \frac{\partial^{4} w}{\partial x^{3} \partial y}+2\left(D_{12}+2 D_{66}\right) \frac{\partial^{4} w}{\partial x^{2} \partial y^{2}}+4 D_{26} \frac{\partial^{4} w}{\partial x \partial y^{3}} \\
& +D_{22} \frac{\partial^{4} w}{\partial y^{4}}+N_{x} \frac{\partial^{2} w}{\partial x^{2}}+2 N_{x y} \frac{\partial^{2} w}{\partial x \partial y}+N_{y} \frac{\partial^{2} w}{\partial y^{2}}=0
\end{aligned}
$$

where the first two equations govern the in-plane resultant loads $\left(u_{0}\right.$ and $v_{0}$ are the in-plane displacements at midplane), and the last equation governs the out-of-plane buckling deflection, $w=w(x, y)$. $N_{x}$ and $N_{y}$ are the in-plane resultant normal loads in the $x$ and $y$ directions, respectively, and $N_{x y}$ is the in-plane shear resultant. $D_{11}$, $D_{12}, D_{16}, D_{22}, D_{26}$, and $D_{66}$ are the flexural rigidities of the plate. The flexural rigidities are obtained from the material constitutive law for each particular finite element.

The boundary conditions for the case of a square plate (side lengths $a \times a$ ) with clamped sides are

$$
\begin{array}{rlrlrl}
w & =0, & \frac{\partial w}{\partial x} & =0 & \text { at } x & =-\frac{a}{2}, \\
w & =0, & & \frac{a}{2} \\
\frac{\partial w}{\partial y} & =0 & \text { at } y & =-\frac{a}{2}, & & \frac{a}{2}
\end{array}
$$

$$
u_{0}, \quad v_{0}=0, \quad \text { at } x, \quad y=-\frac{a}{2}, \quad \frac{a}{2}
$$

The critical increase in temperature at buckling, $\Delta T_{\mathrm{cr}}$, is related to the in-plane resultant loads and homogenized (thickness-averaged) values of thermal expansion, $\bar{\alpha}_{x x}, \bar{\alpha}_{y y}$, and $\bar{\alpha}_{x y}$ by the following equation:

$$
\left[\begin{array}{c}
N_{x} \\
N_{y} \\
N_{x y}
\end{array}\right]=\frac{12 \Delta T_{\mathrm{cr}}}{h^{2}}\left[\begin{array}{lll}
D_{11} & D_{12} & D_{13} \\
D_{21} & D_{22} & D_{23} \\
D_{31} & D_{32} & D_{33}
\end{array}\right]\left[\begin{array}{c}
\bar{\alpha}_{x x} \\
\bar{\alpha}_{y y} \\
\bar{\alpha}_{x y}
\end{array}\right]
$$

A quarter-plate was first analyzed using the finite element algorithm with the assumption of symmetric buckling modes. The plate was modeled using 2888 plate elements. Each plate element has four nodes and five degrees of freedom: $u, v, w, d w / d x$, and $d w / d y$. The general finite element formulation for this buckling problem is based on both the conventional element flexural stiffness matrix $\left[k_{b}\right]$ and geometric element stiffness matrix $\left[k_{g}\right]$ that takes account of the in-plane load resultants. The formulations for $\left[k_{b}\right]$ and $\left[k_{g}\right]$ are given in Eq. (11):

$$
\left[k_{b}\right]=\iiint_{V} B_{2}^{T} D B_{2} \mathrm{~d} V, \quad\left[k_{g}\right]=\iiint_{V} B_{1}^{T} P B_{1} \mathrm{~d} V
$$

where $B_{1}$ and $B_{2}$ are the matrices consisting of the first and second derivatives of the shape functions of the plate element, respectively. The flexural rigidity $D$ is defined in Eq. (4). The matrix $P$ incorporates the in-plane load resultants $N_{x}, N_{y}$, and $N_{x y}$. Based on this formulation, the element stiffness matrix for the buckling problem is given next:

$$
[k]=\left[k_{b}\right] \pm\left[k_{g}\right]
$$

where the sign of the geometric stiffness matrix $\left[k_{g}\right]$ depends on the type of in-plane loads. It is positive if the in-plane loads are tensile, and it is negative if the in-plane loads are compressive. At buckling, the condition given next is satisfied:

$$
\operatorname{det}\left(\left[K_{b}\right] \pm \lambda\left[K_{g}\right]\right)=0
$$

where $\left[K_{b}\right]$ and $\left[K_{g}\right]$ are global flexural and geometric stiffness matrices, respectively. Equation (13) shows that the solution finally leads to an eigenvalue problem. The $\Delta T_{\text {cr }}$ value that satisfies the critical buckling condition is the critical increase in temperature.

\section{Verification of Finite Element Methodology}

Verification of the finite element approach of this work consists of three steps: fringe contour comparison for an isotropic plate problem, stress distribution verification for a unidirectional orthotropic composite laminate, and buckling solution for a benchmark orthotropic plate problem.

\section{A. Fringe Contour Comparison}

The developed finite element methodology is initially verified by comparing the fringe contours for a tension problem where an isotropic plate with a central cutout is subjected to remote tensile loading in the $y$ direction (Fig. 3). This case has been selected because the fringe contours of the theoretical solution are available in the literature [20], and the problem geometry is similar to the buckling problem of interest in this work. The fringe contours plotted for the finite element solution match well with the theoretical result as shown in Fig. $\underline{4}$, providing a degree of confidence in mesh selection.

\section{B. Stress Distribution Verification for a Unidirectional Orthotropic Laminate}

The second check on the finite element mesh quality is to verify the predicted stress concentration factor $(\mathrm{SCF})$ of a four-layer unidirectional orthotropic laminate, with a stacking sequence of 
Theoretical Fringe Contours

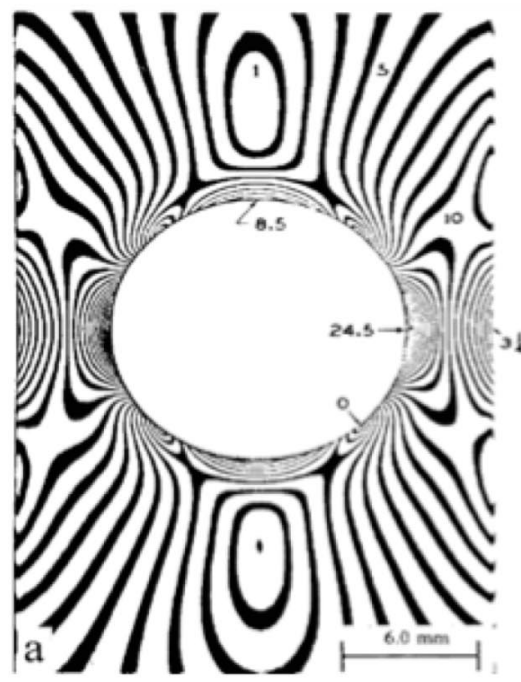

Numerical Fringe Contours

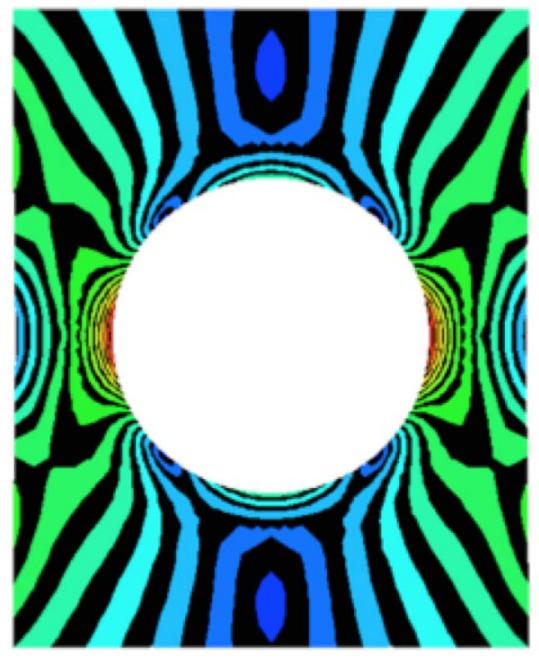

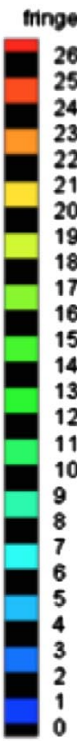

Fig. 4 Fringe contour comparison.

[45/0] s, to the theoretical solution. This problem has the same geometry as the problem of interest in this work, a plate with a central cutout, but in this case, the plate is under the effect of tensile loads acting on the horizontal direction. A detailed explanation about the problem and solution methodology can be found in the earlier work of the authors [9]. The maximum SCF value of the finite element solution was found to be at the cutout boundary, and it is 6.27 . This value is close to the theoretical value, 5.96 [21]

\section{Benchmark Problem for Thermal Buckling of an Orthotropic Plate}

Using the finite element method, the critical increase in temperature was computed for symmetric buckling of a clamped symmetrically laminated plate under uniform heating. However, no analytical solution exists for the corresponding buckling problem. To verify the obtained buckling solution, a similar but simpler case of a specially orthotropic plate, which has a known analytical solution [19], was employed. A rectangular orthotropic plate with principal directions parallel to the sides is compressed by a uniformly distributed edge axial load $P_{x}$ (Fig. 5).

The buckling problem for such a plate with four simply supported sides is given by Lekhnistkii [19]. The buckling equation is

$$
D_{11} \frac{\partial^{4} w}{\partial x^{4}}+2 D_{33} \frac{\partial^{4} w}{\partial x^{2} \partial y^{2}}+D_{22} \frac{\partial^{4} w}{\partial y^{4}}+N_{x} \frac{\partial^{2} w}{\partial x^{2}}+N_{y} \frac{\partial^{2} w}{\partial y^{2}}=0
$$

The solution of the form

$$
w=A_{m n} \sin \left(\frac{m \pi x}{a}\right) \sin \left(\frac{n \pi y}{b}\right)
$$

is sought, where $A_{m n}$ are constant coefficients, and $m$ and $n$ are integers. The following boundary conditions are used:

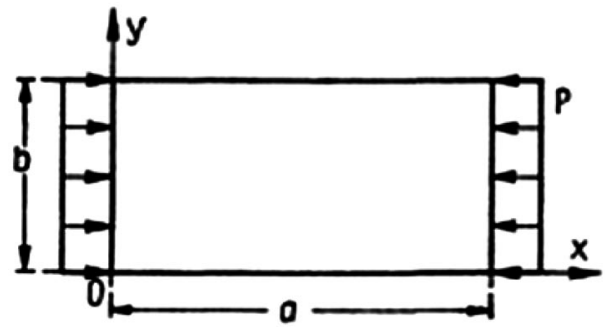

Fig. 5 Benchmark buckling problem definition for an orthotropic plate.

$$
\begin{array}{llrl}
w=0, & \frac{\partial w^{2}}{\partial x^{2}}+\nu_{y} \frac{\partial w^{2}}{\partial y^{2}}=0 & \text { at } x=0, & a \\
w=0, & \frac{\partial w^{2}}{\partial y^{2}}+\nu_{x} \frac{\partial w^{2}}{\partial x^{2}}=0 & \text { at } y=0, & b
\end{array}
$$

where $\nu_{x}$ and $\nu_{y}$ are Poisson's ratios. By requiring that Eq. (15) be a solution of Eq. (14), the following solution is obtained:

$$
N_{x}\left(\frac{m}{a}\right)^{4}+N_{y}\left(\frac{n}{b}\right)^{4}=\pi^{2}\left[D_{11} \frac{m}{a}+2 D_{33} \frac{m n}{a b}+D_{22} \frac{n}{b}\right]
$$

The formulation is performed for the case of proportional loading, where forces $N_{x}$ and $N_{y}$ may vary (general case) but must maintain a constant ratio $\beta$ :

$$
N_{x}=\lambda, \quad N_{y}=\lambda \beta
$$

The critical value of $\lambda$ is found from the following formula:

$$
\begin{aligned}
& \lambda=\frac{\pi^{2} \sqrt{D_{11} D_{22}}}{b^{2}} \\
& . \frac{\sqrt{D_{11} / D_{22}}(m / c)^{2}+\left(2 D_{33} / \sqrt{D_{11} D_{22}}\right) n^{2}+\sqrt{D_{22} / D_{11}}(c / m)^{2} n^{4}}{1+\beta(c / m)^{2} n^{2}}
\end{aligned}
$$

where $c$ is the ratio between the lengths of the sides of the plate $(c=a / b)$. The problem then consists of seeking the values of $m$ and $n$ that give the smallest $\lambda$ and hence the critical buckling load $\lambda_{\mathrm{cr}}$. Based on the solution of the critical buckling load, the expression can be formulated for the critical increase in temperature using the coefficient of thermal expansion tensor $\alpha$ :

$$
\Delta T_{\mathrm{cr}}=\frac{\lambda}{12 / h^{2}\left(D_{11} \cdot \alpha_{x}+D_{12} \cdot \alpha_{y}+D_{13} \cdot \alpha_{x y}\right)}
$$

By modifying the boundary conditions in the original calculations (from clamped to simply supported for an orthotropic plate), the results are compared to the analytical solution and are found to be a very close match. Sample comparisons are shown later. The default orthotropic rectangular plate has dimensions $(a \times b \times h)$ of 
Table 2 Comparison of results for a simply supported rectangular orthotropic plate

\begin{tabular}{lccccc}
\hline \hline$D_{11}, \mathrm{~N} \cdot \mathrm{m}$ & $D_{12}, \mathrm{~N} \cdot \mathrm{m}$ & $\alpha_{x}, K^{-1}$ & $b, \mathrm{~m}$ & Analytical $\Delta T_{\mathrm{cr}}, \mathrm{K}$ & Computed $\Delta T_{\mathrm{cr}}, \mathrm{K}$ \\
\hline $7.0 \times 10^{10}$ & -- & -- & -- & 192.9 & 193.2 \\
$8.0 \times 10^{10}$ & -- & -- & -- & 187.4 & 187.4 \\
$9.0 \times 10^{10}$ & -- & -- & -- & 183.2 & 183.2 \\
-- & $1.5 \times 10^{9}$ & -- & -- & 188.9 & 188.9 \\
-- & $2.0 \times 10^{9}$ & -- & -- & 187.4 & 187.4 \\
-- & $2.5 \times 10^{9}$ & -- & -- & 186.0 & 185.9 \\
-- & -- & $8.0 \times 10^{-6}$ & -- & 209.0 & 209.0 \\
-- & -- & $9.0 \times 10^{-6}$ & -- & 187.4 & 187.4 \\
-- & -- & $10 \times 10^{-6}$ & -- & 169.9 & 169.8 \\
-- & -- & -- & 0.3 & 211.1 & 211.2 \\
-- & -- & -- & 0.4 & 187.4 & 187.4 \\
-- & -- & -- & 0.5 & 171.3 & 171.2 \\
\hline \hline
\end{tabular}

$0.1 \times 0.4 \times 0.004 \mathrm{~m}$. Its flexural rigidities are $D_{11}=8.0 \times 10^{10}$, $D_{12}=D_{21}=2.0 \times 10^{9}, D_{22}=4.0 \times 10^{10}$, and $D_{33}=1.2 \times 10^{11}$, and its coefficients of thermal expansion are $\alpha_{x}=9.0 \times 10^{-6}$ and $\alpha_{y}=1.2 \times 10^{-6}$. Only one property is varied at a time, and the average percentage error between the results is $0.0314 \%$. The analytical and computational critical increases in temperature are presented in Table 2 .

\section{Optimization of Fiber Path}

The optimization problem deals with multiple objectives because the goals are defined to find the fiber path and layer thicknesses that maximize the critical increase in buckling temperature $\Delta T_{\mathrm{cr}}$ and minimize the mass of the structure as well. The fiber path depends on four independent variables for each layer: $T_{i, 1}^{q}, T_{i, 2}^{q}, T_{i, 3}^{q}$, and $T_{i, 4}^{q}$. All $T_{f, p}^{q}$ values have $T_{i, p}^{q}$ equivalents, and $d \theta_{p}^{q}$ can also be computed in terms of them. Considering the fact that the problem of interest includes a four-layer symmetric laminate and two independent layers, the number of optimization parameters relevant to fiber path distribution is eight. The remaining optimization variables are the thicknesses of these independent two layers $\left(z_{1}\right.$ and $\left.z_{2}\right)$ because the area is constant for every layer, and therefore the total mass varies with respect to the thicknesses. A multi-objective optimization problem is defined because proposing only one objective that is related to buckling may cause designs that are not cost efficient. To eliminate this possibility, minimization of the plate mass is defined as the second design objective. Because the distribution constraints were already imposed to the problem through meshing and methodology, no additional design constraints are defined for the optimization problem. The mathematical formulation of the optimization problem is given in Eq. (21):

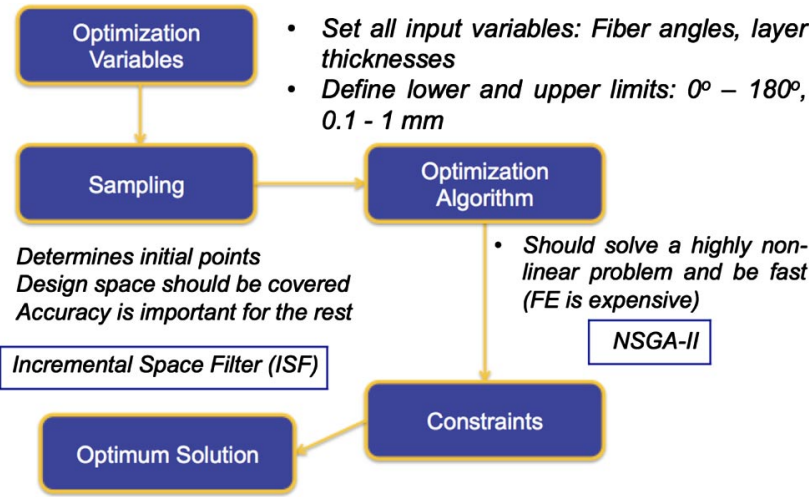

Fig. 6 Optimization workflow.

\begin{tabular}{lc}
\multicolumn{2}{c}{$\begin{array}{c}\text { Table 3 } \\
\text { design parameters }\end{array}$} \\
\hline \hline Parameter & Optimum value \\
\hline$T_{i_{1}}^{1}$ & $5.5571 \mathrm{deg}$ \\
$T_{i_{2}}^{1}$ & $179.99 \mathrm{deg}$ \\
$T_{i_{3}}^{1}$ & $2.7640 \mathrm{deg}$ \\
$T_{i_{4}}^{1}$ & $1.9010 \mathrm{deg}$ \\
$T_{i_{1}}^{2}$ & $2.2260 \mathrm{deg}$ \\
$T_{i_{2}}^{2}$ & $6.0188 \mathrm{deg}$ \\
$T_{i_{3}}^{2}$ & $89.9840 \mathrm{deg}$ \\
$T_{i_{4}}^{2}$ & $1.9855 \mathrm{deg}$ \\
$z_{1}$ & $0.10085 \mathrm{~mm}$ \\
$z_{2}$ & $0.10120 \mathrm{~mm}$ \\
$\Delta T_{\mathrm{cr}}$ & $205.75^{\circ} \mathrm{C}$ \\
\hline \hline
\end{tabular}

$$
\begin{aligned}
& \max f_{1}=\Delta T_{\mathrm{cr}}, \quad \min f_{2}=m\left(z_{1}, z_{2}\right) \\
& \quad s=\left(T_{i_{1}}^{1}, T_{i_{2}}^{1}, T_{i_{3}}^{1}, T_{i_{4}}^{1}, T_{i_{1}}^{2}, T_{i_{2}}^{2}, T_{i_{3}}^{2}, T_{i_{4}}^{2}, z_{1}, z_{2}\right) \\
& 0 \leq T_{i_{1}}^{1}, T_{i_{2}}^{1}, T_{i_{3}}^{1}, T_{i_{4}}^{1}, T_{i_{1}}^{2}, T_{i_{2}}^{2}, T_{i_{3}}^{2}, T_{i_{4}}^{2}<180 \mathrm{deg} \\
& 0.1 \mathrm{~mm} \leq z_{1}, \quad z_{2} \leq 1 \mathrm{~mm}
\end{aligned}
$$

In this formulation, the first line shows the objective functions where $m$ shows the mass of the plate. The set of optimization variables is defined in the second line. The upper and lower limits of the optimization variables are given in the last two lines. Because the problem of interest here is a nonlinear multi-objective optimization problem, a genetic algorithm that is also capable of working with multiple objectives is implemented. Considering the large computational time requirement for a finite element buckling solution, an efficient optimization algorithm needs to be chosen. For this purpose, the nondominated sorting genetic algorithm II (NSGA-II), which is known to be one of the fastest genetic algorithms, was implemented. The sampling was performed with an incremental space filter (ISF) with 10 designs of experiments (DOEs). The optimization algorithm provided 150 total designs because it used 15 function evaluations for each DOE. The summary of the optimization workflow is illustrated in Fig. 6.

The parameters of the optimum design are shown in Table 3. The optimum design performance is compared to selected random designs in Table 4 . These random designs have the same layer thickness values as the optimum design. However, their layers are defined as unidirectional laminas. The comparison indicates that the optimum design has provided a significant increase in the thermal buckling performance of the composite structure having the same mass. The fiber path distributions of the optimum design for layers 1 and 2 are illustrated in Figs. $\underline{7}$ and $\underline{8}$, respectively. 
Table 4 Performance comparison for optimum design

\begin{tabular}{llllllllll}
\hline \hline Design & $T_{i_{1}}^{1}, \operatorname{deg}$ & $T_{i_{2}}^{1}, \operatorname{deg}$ & $T_{i_{3}}^{1}, \operatorname{deg}$ & $T_{i_{4}}^{1}, \operatorname{deg}$ & $T_{i_{1}}^{2}, \operatorname{deg}$ & $T_{i_{2}}^{2}, \operatorname{deg}$ & $T_{i_{3}}^{2}, \operatorname{deg}$ & $T_{i_{4}}^{2}, \operatorname{deg}$ & $\Delta T_{\text {cr }},{ }^{\circ} \mathrm{C}$ \\
\hline Random 1 & 45 & 45 & 45 & 45 & 0 & 0 & 0 & 0 & 151.68 \\
Random 2 & 90 & 90 & 90 & 90 & 135 & 135 & 135 & 135 & 121.42 \\
Optimum & 5.5571 & 179.99 & 2.7640 & 1.9010 & 2.2260 & 6.0188 & 89.9840 & 1.9855 & 205.75 \\
\hline \hline
\end{tabular}

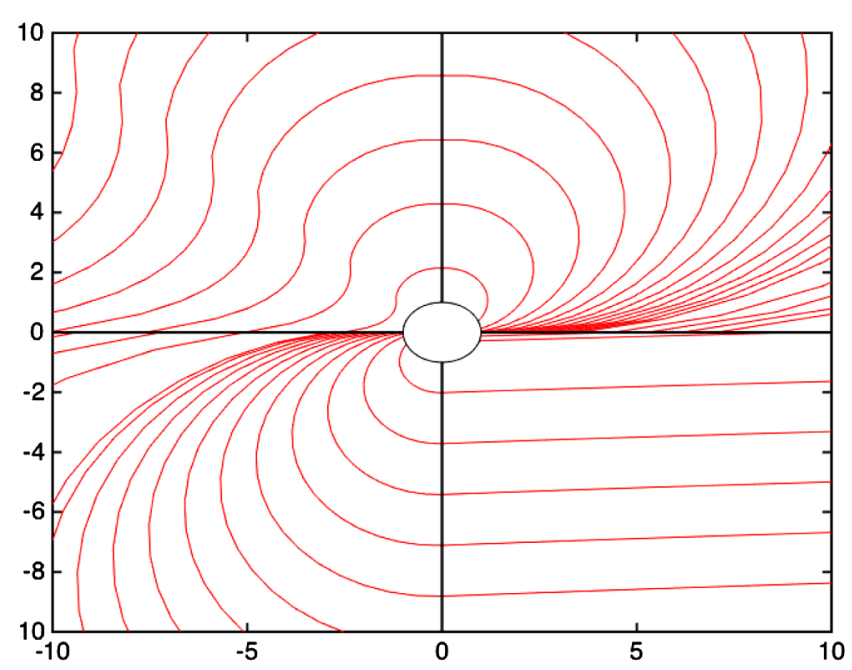

Fig. 7 Optimum fiber path (layers 1 and 4).

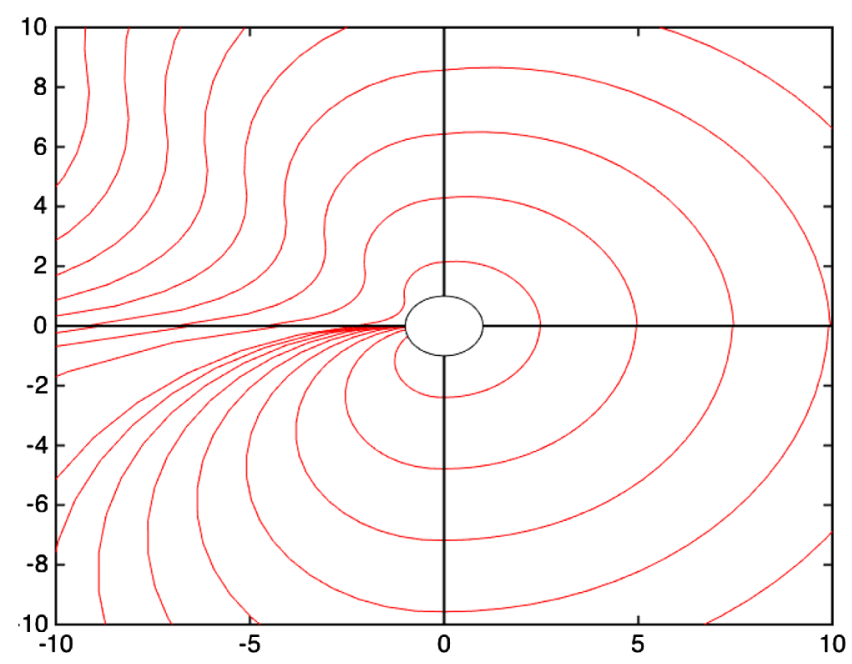

Fig. 8 Optimum fiber path (layers 2 and 3).

\section{Conclusions}

A computationally efficient and novel finite element approach to identify optimum steered fiber paths that can be manufactured using AFP technology is presented in this work. The meshing algorithm using discrete fiber angle values within each element as control variables for each independent layer is developed so as to obtain continuous fiber paths as well as to reduce the computational time. The specific problem discussed here addresses investigating optimal steered fiber paths for an orthotropic symmetric laminate under uniform thermal loads to maximize the critical buckling temperature. The plate has a cutout at its center and is clamped on all sides. A full plate model is developed to include symmetric and asymmetric buckling modes. The laminate is modeled in four separate quadrants, using finite elements, ensuring continuity of displacements and fiber angles across the quadrants. A verification of the algorithm is provided by three specific cases: a fringe contour comparison for an isotropic plate that is available in the literature, an orthotropic plate tension problem using unidirectional plies, and a benchmark buckling problem for orthotropic laminates with unidirectional plies. After the verification of the solution methodology, the global optimization is performed using a genetic algorithm using the defined objective functions and the optimization parameters. The optimum design is selected among all feasible solutions, and it provides a very significant increase in the thermal buckling capacity.

\section{References}

[1] Cemenska, J., Rudberg, T., and Henscheid, M., "Automated In-Process Inspection System for AFP Machines," SAE International Journal of Aerospace, Vol. 8, No. 2, 2015, pp. 303-309.

doi: $10.4271 / 2015-01-2608$

[2] Kirsch, G., "The Theory of Elasticity and the Needs of the Strength of Materials," Zeitschrift des Vereines Deutscher Ingenieure, Vol. 42, No. 29, 1898, pp. 797-807.

[3] Mansfield, E. H., "Neutral Holes in Plane Sheet-Reinforced Holes Which Are Elastically Equivalent to the Uncut Sheet," Quarterly Journal of Mechanics and Applied Mathematics, Vol. 3, No. 3, 1953, pp. $370-378$. doi:10.1093/ajmam/6.3.370

[4] Pipes, R. B., Wetherhold, R. C., and Gillespie, J. W., Jr., "Notched Strength of Composite Materials," Journal of Composite Materials, Vol. 13, April 1979, pp. 148-160. doi: $10.1177 / 002199837901300206$

[5] Tang, S., "Interlaminar Stresses Around Circular Cutouts in Composite Plates Under Tension," AIAA Journal, Vol. 15, No. 11, 1977, pp. 16311637. doi: $10.2514 / 3.60828$

[6] Ahn, J. H., and Waas, A. M., "Prediction of Compressive Failure in Laminated Composites at Room and Elevated Temperature," AIAA Journal, Vol. 40, No. 2, 2002, pp. 346-358.

[7] Senocak, E., and Waas, A. M., "Design Considerations for Symmetrically Laminated Plates with Cutouts," 33rd Structures, Structural Dynamics and Materials Conference, AIAA Paper 1992$2486,1992$. doi: $10.2514 / 6.1992-2486$

[8] Senocak, E., and Waas, A. M., "Neutrally Reinforced Holes in Symmetrically Laminated Plates," Journal of Aircraft, Vol. 30, No. 3, 1993, pp. 428-430. doi: $10.2514 / 3.46360$

[9] Acar, P., Vijayachandran, A., Sundararaghavan, V., Waas, A. M., and Rassaian, M., "Optimization of Spatially Varying Fiber Paths for a Symmetric Laminate with a Circular Cutout Under Remote Uniaxial Tension," SAE International Journal of Materials and Manufacturing, Vol. 9, No. 1, 2016, pp. 75-80. doi:10.4271/2015-01-2609

[10] Lin, C. C., and Kuo, C. S., "Buckling of Laminated Plates with Holes," Journal of Composite Materials, Vol. 23, No. 6, 1989, pp. 536-553. doi:10.1177/002199838902300601

[11] Topal, U., and Uzman, U., "Maximization of Buckling Load of Laminated Composite Plates with Central Circular Holes Using MFD Method," Structural and Multidisciplinary Optimization, Vol. 35, No. 2, 2008, pp. 131-139. doi:10.1007/s00158-007-0119-1

[12] Ounis, H., Tati, A., and Banchabane, A., "Thermal Buckling Behavior of Laminated Composite Plates: A Finite-Element Study," Frontiers of Mechanical Engineering, Vol. 9, No. 1, 2014, pp. 41-49. doi:10.1007/s11465-014-0284-z

[13] Rangarajan, A., D’Mello, R. J., Sundararaghavan, V., and Waas, A. M., "Minimization of Thermal Expansion of Symmetric, Balanced, Angle Ply Laminates by Optimization of Fiber Path Configurations," Composites Science and Technology, Vol. 71, No. 8, 2011, pp. 11051109.

[14] Hyer, M. W., and Lee, H. H., "The Use of Curvilinear Fiber Format to Improve Buckling Resistance of Composite Plates with Central Circular Holes," Composite Structures, Vol. 18, No. 1, 1991, pp. 239-261. doi:10.1016/0263-8223(91)90035-W 
[15] Gurdal, Z. W., and Olmedo, R., "In-Plane Response of Laminates with Spatially Varying Fiber Orientations: Variable Stiffness Concept," AIAA Journal, Vol. 31, No. 4, 1993, pp. 751-758.

[16] DeTeresa, S. J., Allison, L. M., Freeman, D. C., and Groves, S. E., "Matrix-Dominated Performance of Thick-Section Fiber Composites for Flywheel Applications," 46th International Society for Advancement of Material and Process Engineering (SAMPE) Symposium, Long Beach, CA, May 2001.

[17] O'Brien, T. K., Composite Materials: Fatigue and Fracture, ASTM International, Philadelphia, PA, 1991, p. 35.

[18] Duran, A. V., Fasanella, N., Sundararaghavan, V., and Waas, A. M., "Optimization of Composite Plates with Spatially Varying Fiber Paths for Thermal Buckling," 56th AIAA/ASCE/AHS/ASC Structures, Structural Dynamics, and Materials Conference, AIAA Paper 2015$0454,2015$.

doi:10.2514/6.2015-0454

[19] Lekhnitskii, S. G., Anisotropic Plates, Gordon and Breach, New York, 1968, pp. 445-459.

[20] Frocht, M. M., Photoelasticity, Vol. 1, Wiley, New York, 1941, pp. 77-89.

[21] Toubal, L, Karama, M., and Lorrain, B., "Stress Concentration in a Circular Hole in Composite Plate," Composite Structures, Vol. 68, No. 1, 2005, pp. 31-36.

doi:10.1016/j.compstruct.2004.02.016 